\title{
The Body Plan Concept and Its Centrality in Evo-Devo
}

\author{
Katherine E. Willmore
}

Published online: 14 June 2012

(C) Springer Science+Business Media, LLC 2012

\begin{abstract}
A body plan is a suite of characters shared by a group of phylogenetically related animals at some point during their development. The concept of bauplane, or body plans, has played and continues to play a central role in the study of evolutionary developmental biology (evo-devo). Despite the importance of the body plan concept in evodevo, many researchers may not be familiar with the progression of ideas that have led to our current understanding of body plans, and/or current research on the origin and maintenance of body plans. This lack of familiarity, as well as former ties between the body plan concept and metaphysical ideology is likely responsible for our underappreciation of the body plan concept in its own right, as well as its role in evo-devo. My aim in this review is to outline how we have arrived at our modern definition of body plan, the controversies associated with the concept, its role in evodevo, and how current research is informing us on body plans. To this end, I integrate concepts such as the nature of phyla, the Cambrian explosion, constraint, evolvability, and results from recent research on gene regulatory networks with the much older concept of the body plan.
\end{abstract}

Keywords Body plan · Bauplan · Constraint · Evolvability · Evolution · Development

The concept of bauplane, or body plans, has played and continues to play a central role in the study of evolutionary developmental biology (evo-devo). Bauplan (plural, bauplane) as a term used in biology was first introduced

\section{K. E. Willmore $(\triangle)$}

Department of Anthropology, Pennsylvania State University, 409 Carpenter Building, University Park,

State College, PA 16802, USA

e-mail: katwillmore@gmail.com by Joseph Henry Woodger in 1945, and means ground plan or structural plan (Hall 1999; Rieppel 2006; Woodger 1945). Essentially, a body plan is a suite of characters shared by a group of phylogenetically related animals at some point during their development. However, long before the term body plan was coined, its importance was demonstrated in research programs that presaged the field of evo-devo, perhaps most famously (though erroneously) by Ernst Haeckel's recapitulation theory. Since the rise of evo-devo as an independent field of study, the body plan concept has formed the backbone upon which much of the current research is anchored. The body plan concept is explicit in studies of homology, modularity and integration, canalization, key innovations, heterochrony, variability, evolvability, and arguably all aspects of modern evo-devo research.

While I argue that the body plan concept is central to all evo-devo research, its importance is often undervalued or disputed. Many researchers may not be familiar with the progression of ideas that have led to our current understanding of body plans, and/or current research on the origin and maintenance of body plans. This lack of familiarity, as well as former ties between the body plan concept and metaphysical ideology is likely responsible for our underappreciation of the body plan concept in its own right, as well as its role in evo-devo. The importance of the body plan concept in evo-devo research is not a new argument (see Atkinson 1992; Hall 1996, 1999). However, our current concept of the body plan is still largely misunderstood, and I therefore suggest that a review of the body plan concept is worthwhile. Here, my aim is to outline how we have arrived at our modern definition of body plan, the controversies associated with the concept, its role in evo-devo, and how current research is informing us on body plans. To this end, I integrate concepts such as the nature of phyla, the Cambrian explosion, constraint, evolvability, and results from recent 
research on gene regulatory networks with the much older concept of the body plan.

\section{A Historical Look at the Body Plan Concept}

The body plan concept has a long history, with many key players adding their own twists to the idea. The path to our current concept is rather tortuous, with aspects left behind as relics by some scholars only to be picked up again later by others. Some of these renewed ideas were best left in the past, while others have largely informed our current understanding of body plans. To untangle the confusion and controversies surrounding the body plan concept, it is necessary to visit its history (see Table 1 for a historical summary).

\section{Aristotle}

As with most concepts in biology, roots for the body plan concept can be traced back to Aristotle. He described the unity of plan, which is essentially a structural plan shared by a group of organisms that he used to classify animals (Hall 1999; Mayr 1982). Aristotle's unity of plan and classification system is based on the scala naturae (ladder of nature), whereby humans are the ideal to which all other animal structures are related.

\section{Buffon}

Buffon (1707-1788) introduced the concept of the unity of the type, whereby animals descended from a small number of types or possibly from a single type (Hall 1999). This concept of form initiated the idealistic or transcendental school of biology, where the form of animals is considered an expression of an idealized archetype.

\section{Cuvier}

Following from Buffon's idealistic school of morphology, Georges Cuvier (1769-1832) divided the animal kingdom into four discrete groups or embranchments. These embranchments were essentially equivalent to what we now call phyla and were based on four different types of nervous system (Hall 1996, 1999; Panchen 2001). Cuvier's classification system based on the principle of the correlation of parts directly reflects his assertion that function determines form (Mayr 1982). The correlation of parts is based on functional relationships among organisms, such that suites of features were always grouped together. These suites of characters ensure that the animal is perfectly adapted to its environment. A necessary consequence of Cuvier's principle of the correlation of parts is that transformation between the four types is impossible, as the correlation of structures required for life would be disrupted in such intermediate organisms (Hall 1996, 1999; Mayr 1982; Panchen 2001).

\section{Geoffroy}

Etienne Geoffroy Saint Hilaire (1722-1844) was diametrically opposed to his colleague Cuvier in terms of morphology and classification. Where Cuvier asserted that function determines form, Geoffroy maintained that form determines function. Additionally, instead of Cuvier's separate ground plan for each embranchment, Geoffroy attempted to fit all organisms into a single structural plan (Appel 1987; Hall 1999; Mayr 1982).

Later in his career, Geoffroy and his protégé Etienne Serres used experimental embryology to found a new branch of research called teratology (the study of developmental abnormalities, Hall 1999; Panchen 2001). Geoffroy used his findings from his teratology research as evidence for the maintenance of the basic structural plan even when organisms are exposed to environmental influences that are expected to change this plan (Hall 1999). Also, from this research, the theory of recapitulation was born. Features of "higher" vertebrates arrested in development after exposure to teratogens (chemicals or environmental factors that cause developmental abnormalities) were equivalent to normal developmental stages in "lower" vertebrates (Hall 1999). This embryological relationship among vertebrates was

Table 1 Summary of ideas from the early key players that have shaped our current concept of the body plan

\begin{tabular}{|c|c|c|c|}
\hline Aristotle & $384-322 \mathrm{BC}$ & Unity of plan & Describes the structural plan used to classify animals based on the ladder of nature \\
\hline Buffon & $1707-1788$ & Unity of the type & States that animal forms descended from an idealized archetype \\
\hline Cuvier & $1769-1832$ & Function determines form & Divides animals into groups based on the function of their anatomical traits \\
\hline Geoffroy & $1722-1844$ & Form determines function & Demonstrates the relationships among animals based on the similarities of form \\
\hline von Baer & $1792-1876$ & $\begin{array}{l}\text { Embryological concept of } \\
\text { body plan }\end{array}$ & $\begin{array}{l}\text { Based on his four laws of development, von Baer showed that types reflect } \\
\text { the structural organization of embryos rather than that of adults }\end{array}$ \\
\hline Owen & $1804-1892$ & Archetype & Returns to an animal classification system based on a divine, idealized form \\
\hline Darwin & $1809-1882$ & $\begin{array}{l}\text { Descent from a common } \\
\text { ancestor }\end{array}$ & $\begin{array}{l}\text { Replaced the common archetype used to define the body plan concept with } \\
\text { common ancestry }\end{array}$ \\
\hline Haeckel & $1834-1919$ & Biogenetic law & $\begin{array}{l}\text { Concept that individual development repeats the evolutionary history of the adult } \\
\text { morphology of its ancestors }\end{array}$ \\
\hline
\end{tabular}


more thoroughly elaborated on by von Baer and was reinterpreted and taken to the extreme by Haeckel.

\section{von Baer}

Karl von Baer (1792-1876) led the transition from the adult archetype to the embryological type. That is, types reflect the structural organization of embryos rather than that of adults (Hall 1996). This switch to the embryological type represented a major transition in morphological research and, as Hall (1999) argues, laid the foundation of modernday evolutionary developmental biology.

The views of von Baer (1828) on the relationships between animals are outlined by his four laws of development. Hall (1999) summarizes these laws as follows:

1. The more general characters of the group to which an embryo belongs appear in development before the more specialized characters;

2. Less general structures form in development after more general structures, until finally the most specialized structures appear;

3. During development, embryos progressively diverge from embryos of other groups; and

4. Embryos of higher animals resemble embryos and not adults of other animals.

These four laws require that embryological criteria are used for classification. Thus, in order to understand phylogenetic relationships, one must understand ontogenetic relationships (Hall 1996). The four laws of von Baer largely foreshadow our current body plan concept; all that was required was the replacement of the archetype with the ancestral form (Hall 1999).

\section{Owen}

In the tradition of Buffon's idealistic morphology, Richard Owen (1804-1892) coined the term archetype in relation to the body plan in 1848 (Hall 1999; Raff 1996). Owen asserted that the archetype was a basic anatomical design shared by all animals within a phylum. Contrary to our current body plan concept, Owen's archetype reflected a divine, idealized form that determines the fundamental traits of animals within a phylum and limits possible variation (Hall 1999; Raff 1996). Our current idea of body plan represents evolutionarily shared and modified traits and would have to wait for Darwin's theory of evolution.

\section{Darwin}

With Darwin's theory of descent from a common ancestor came the realization that groups of animals share a common body plan because they share a common ancestor. That is, common ancestors replaced common archetypes to explain similar body plans (Hall 1999; Mayr 1982). As such, the body plan concept transitioned from an idealistic to a materialistic concept.

The link between the archetype and common ancestry was immediately apparent after publication of The Origin, as Darwin used similarities among embryonic structures as evidence for his theory of common descent. In fact, von Baer's laws influenced Darwin profoundly, particularly the role of embryology (ontogeny) in explaining phylogeny. While Darwin recognized the important connections between ontogeny and phylogeny, it was Haeckel who through his reinterpretation of Darwin's and von Baer's ideas took this connection furthest.

\section{Haeckel}

Ernst Haeckel (1834-1919) coupled Darwin's theory that all animals have descended from a common ancestor with von Baer's concept that embryos represent the archetype and his observation that early vertebrate embryos look alike, to propose his biogenetic law (Haeckel 1874). Essentially, the biogenetic law (or theory of recapitulation) states that individual development repeats the evolutionary history of the adult morphology of its ancestors (Gould 1977). The consequence of the biogenetic law was that evolutionary history is recorded in the embryo phenotype, and therefore, one could find ancestral forms by simply studying the embryos of living organisms (Hall 1999).

Using vertebrate development, Haeckel identified eight specific stages, with each developmental stage representing an ancestral stage of morphology. From these stages came one of the most iconic images of evo-devo, featured in Haeckel's publication Anthropogenie (1874), as discussed in Richardson et al. (1997; Fig. 1). Unfortunately for the body plan concept, and for evo-devo as a field of study, Haeckel's biogentic law and his simplistic and convincing figures depicting similarities between vertebrate embryos created a popular biological movement whereby advocates were referred to as recapitulationists. The simplicity of using embryonic forms to reconstruct phylogenetic trees proved enticing to researchers in the early years following publication of Darwin's Origin. In fact, even Darwin and Huxley were mild recapitulationists (Gould 1977). However, by the end of the nineteenth century, Haeckel's recapitulation theory came under strong criticism (Gould 1977; Mayr 1982; Richardson et al 1997). Researchers increasingly realized that embryos of "higher" animals resemble the embryos, and not adults of "lower" animals as von Baer had stated in 1828 in his fourth law of development. Additionally, some researchers argued that Haeckel glossed over variation between early vertebrate embryos, suggesting that his drawings were inaccurate (Lillie 1919; Sedgwick 1894). 
Fig. 1 Haeckel's famous depiction of his biogenetic law, or recapitulation theory. The upper row is representative of early development, the middle row of mid-development, and the lower row of late development. Using the labels used by Haeckel, the embryos represented include: $F$ fish, $A$ salamander, $T$ turtle, $H$ chick, $S$ pig, $R$ cow, $K$ rabbit, and $M$ human. Figure from Anthropogenie by Haeckel (1874)

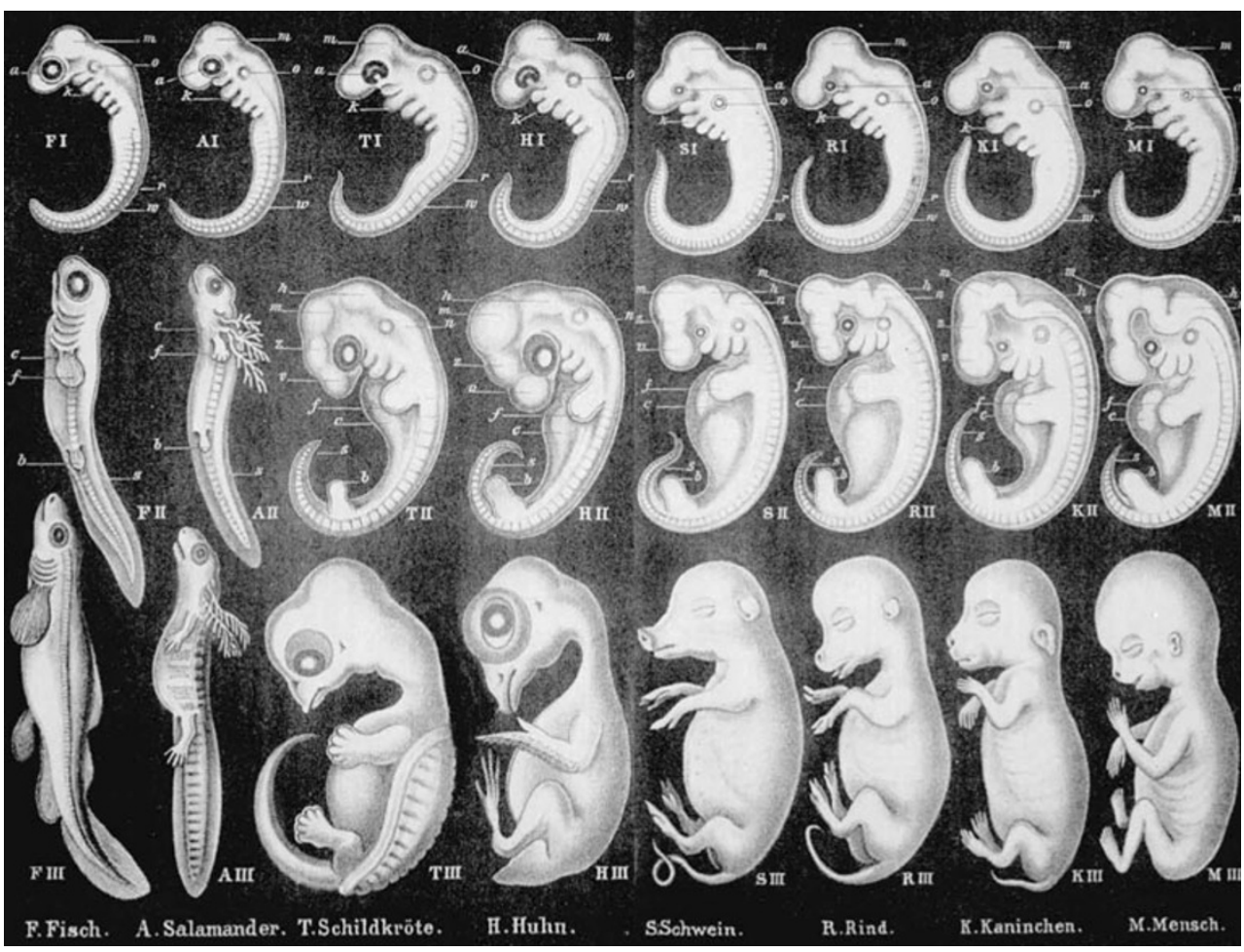

Richardson's thorough analysis of comparative embryology provides evidence that Haeckel's drawings are idealistic representations of embryos, falsely highlighting similarities and ignoring differences among animals (Richardson 1995).

The high profile of Haeckel's biogenetic law ensured that its demise was equally public, and with it went von Baer's much more conservative body plan concept. Rejection of Haeckel's recapitulation theory was complete and so vehement as to essentially make any mention of body plans taboo (Gould 1977). The tarnish created from Haeckel's largely imaginative ideas would remain a blight to evodevo research until the middle-to-late twentieth century and continues to influence many biologists to this day (de Beer 1940, 1958; Garstang 1922; Gould 1977; Hall 1996).

Our current concept of the body plan reflects its long history. As mentioned earlier, Woodger (1945) introduced the term bauplan in 1945. Bauplan is German for building plan or blueprint, and it is based on von Baer's position that the most basic characters develop early in embryonic life (Hall 1999). The generally accepted current concept of the body plan represents the basic anatomical plan shared by higher taxa at the level of the phylum, order, or class (Eldredge 1989; Gould 1989; Hall 1999; Raff 1996; Valentine 1986). For evo-devo, the body plan shared by members of each phylum represents a common pattern of development (Hall 1999; Raff 1996). Thus, in evo-devo, the study of body plans is focused on the origin and maintenance of the developmental programs shared by members within a phylum.

\section{What Are Phyla?}

Given that our current concept of the body plan is tightly associated with phyla, it is important that we have a clear understanding of what phyla are and what they represent. Essentially, phyla represent a taxonomic level or grouping of organisms. In taxonomy, the most inclusive level of organization is found within kingdoms, followed by phyla, classes, orders, families, genera, and, finally, species. There are five recognized kingdoms: Plantae, Animalia, Fungi, Protista, and Monera. Most discussions of body plans are based on phyla within the animal kingdom. Plant body plans are also recognized, but in plants, unlike in animals, there is no fixed developmental trajectory (Cronk, personal communication). Due to space constraints, this discussion is focused on animal body plans and phyla. For readers interested in plant body plans, I recommend Cronk (2001) and Graham et al. (2000).

To be placed within a specific phylum, animals must possess a specific suite of anatomical features (body plan) and they must be monophyletic (share a common ancestor, Nielsen 2003). There are approximately 35 living animal phyla, though opinions differ on the exact number (Raff 1996). Examples of animal phyla include chordates (to which humans belong), echinoderms, mollusks, arthropods, annelids, sponges, and corals, to name a few.

Adult animals do not have to look similar to be placed within the same phylum. Rather, they must share a specific set of anatomical features (body plan) at some point during their life cycle. This reasoning is based on von Baer's fourth 
law that embryos of higher animals resemble embryos, and not adults, of other animals (von Baer 1828). The chordates offer an example of this concept that includes animals with which most of us are familiar. Fish, birds, and mammals (including humans) are all chordates, yet as adults, their appearance is vastly different. To be considered a chordate, animals must possess the following suite of characters: a notochord, pharyngeal slits, a hollow dorsal nerve cord, an endostyle, and a postanal tail (Rychel et al. 2006, Fig. 2).

The notochord is a stiff cellular rod that extends along the length of the body, providing structural support (Carlson 2009, p. 90). In humans, the notochord eventually forms the inner portion of the disks between the vertebrae (Moore and Persaud 2008, p. 62). The pharyngeal slits are clefts found on either side of the throat. In fish, these slits become gills, but in humans, these slits mostly disappear except for one on each side that forms the tympanic membrane or eardrum (Carlson 2009, p. 318). The dorsal nerve cord extends the length of the body adjacent to the notochord and forms the spinal cord in humans and other vertebrates. The endostyle is a groove in the ventral surface of the pharynx or the upper portion of the throat. In humans, the endostyle is thought to form the thyroid gland (Kardong 2006, p. 52). And finally, the postanal tail is just that - a tail found posterior to the anus. As humans, our tail is much reduced as the short coccyx portion of the spine.

As can be seen from our chordate examples, most of the characters that define the chordate body plan are transitory and are only present during a brief portion of the embryonic period. This period of similarity between vastly different animals was first noted by von Baer after a mix-up in his lab, leading to his four laws. "In my possession are two little embryos in spirit, whose names I have omitted to attach and at present I am quite unable to say what class they belong. They may be lizards, or small birds, or very young mammalia, so complete is the similarity in the mode of formation of the head and trunk of these animals" (from Hazkani-Covo et al. 2005, p. 150-151).

\section{What Is the Phylotypic Stage and How Does It Relate to the Developmental Hourglass Model?}

This conserved period during embryogenesis has been called the phylotypic stage as it represents a point in

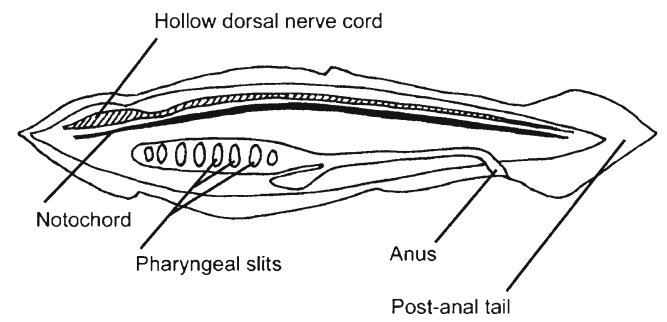

Fig. 2 Body plan characteristics of a stylized chordate development of greatest resemblance among members of a phylum (Ballard 1981; Duboule 1994; Hall 1997, 1999; Raff 1996; Sander 1983; Slack et al. 1993; Wolpert 1991). The phylotypic stage has been most thoroughly studied in insects and vertebrates. In insects, this conserved stage was first called Körpergrundgestalt, or the form-building stage by Seidel (1960, as discussed in Hall 1997). Sander (1983) identified this form-building stage with the germ band stage in insects, a larval stage that includes a head, thorax, and abdomen that are already segmented. Consensus among researchers on the timing of the phylotypic stage in vertebrates is not as great as that for insects. Ballard (1981) defines the phylotypic stage as the pharyngula stage, the point in development just following the appearance of the pharyngeal pouches. Slack et al. (1993) suggest that the tailbud stage in vertebrates represents the phylotypic stage. Wolpert (1991) defines the phylotypic stage at the early somite stage following neurulation, whereas Duboule (1994) broadens the stage to encompass the developmental period between the head fold stage and the tailbud stage.

Additionally, researchers noticed that von Baer's third law (that embryos of different species progressively diverge from one another during ontogeny) does not apply during the earliest stages of development (Elinson 1987; Sander 1983; Seidel 1960). Many examples of early developmental diversity exist, such as the various forms of cleavage and blastula formation in vertebrates, discoidal or spiral cleavage in cephalopods, and the differences between direct and indirect developing echinoderms to name a few (Hall 1996; Raff 1996). Therefore, while many researchers argue that a phylotypic stage does exist, its occurrence is middevelopment, and species arrive at this conserved stage via dramatically different routes (Hall 1999; Newman 2011; Raff 1996). This morphologically conserved intermediate stage of development, preceded and succeeded by developmental diversity, has been called the developmental hourglass (Duboule 1994; Hall 1997, 1999; Raff 1996). The hourglass metaphor depicts a constricted middle section, where development is conserved with the wider portions of the hourglass representing greater variation early and late in development (Fig. 3).

Given the difficulties in defining the phylotypic stage in vertebrates, several researchers have questioned its utility in evo-devo (Bininda-Emonds et al. 2003; Richardson 1995; Richardson et al. 1997; Roux and Robinson-Rechavi et al. 2008). Both morphological and molecular studies of embryonic development have led to conflicting results regarding the timing and existence of the phylotypic stage.

For example, after comparing the external embryonic morphologies of 39 species of vertebrates, including representatives from agnathans, cartilaginous fishes, bony fishes, amphibians, reptiles, birds, and mammals, Richardson et al. (1997) concluded that there is no vertebrate phylotypic 


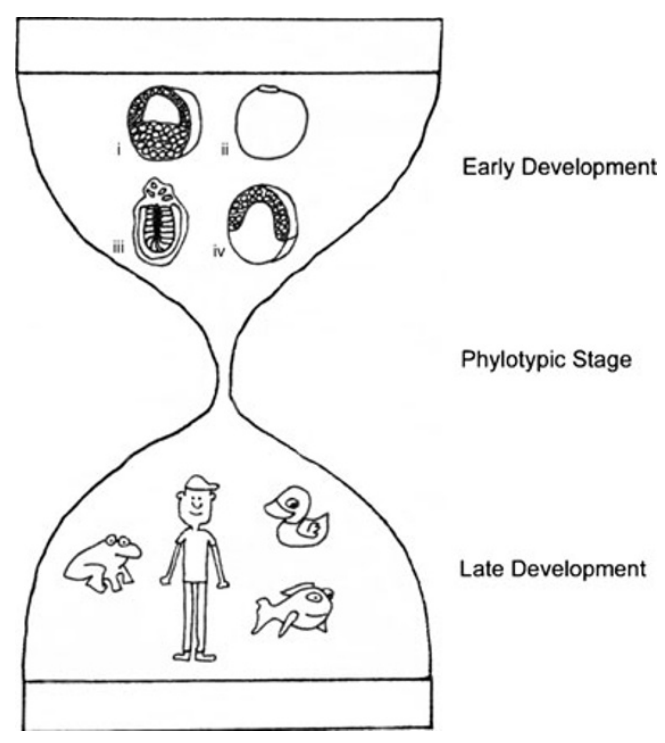

Fig. 3 Illustration of the developmental hourglass model for chordates, specifically vertebrates. The upper portion represents early development, the lower portion late development, and the middle constricted portion the phylotypic stage. The greater width of the upper and lower portions represents the greater morphological diversity that is representative of vertebrates during these developmental periods. For example, the figures in the upper half of the hourglass depict stylized vertebrate embryos and highlight the differences between different species at the beginning of gastrulation. Representative embryos include: $i$ frog, $i i$ bird, $i i i$ human, and $i v$ fish. The lower half depicts the developmental differences that occur late in development that lead to the vast differences we see among adult vertebrate species. The middle constricted portion illustrates the relative lack of morphological variation between vertebrate species during the phylotypic stage

stage. They noted the challenges of defining a common reference stage that applied to all the species they observed, as common markers used to determine the phylotypic stage differed between the species they studied. The tailbud stage described by Slack et al. (1993) was found to be a promising candidate for the phylotypic stage, as it approximates the end of somite segregation in the trunk region, and it was this stage that these authors used for comparisons. However, the anterior structures in marsupials and monotremes are more advanced than other vertebrate species at the tailbud stage. Additionally, somite number varied dramatically at the tailbud stage, ranging from 11 in the Puerto Rican tree frog to over 60 in the blind worm. Tailbud embryos also varied in size, with the scorpion fish measuring only 700 micrometers and the mudpuppy measuring 9.25 millimeters. They also noted several examples of heterochrony (differential timing of development) at the tailbud stage. For example, in amniotes, the heart has completed looping at the tailbud stage, whereas in zebrafish it has not yet begun to form. Given the morphological diversity among the vertebrates they studied, Richardson et al. (1997) argued that the phylotypic stage does not exist and should perhaps more appropriately be referred to as the phylotypic period.
Another group looked at variation in the timing of developmental events among vertebrates as a whole, and mammals alone, to test the validity of the hourglass model (Bininda-Emonds et al. 2003). Specifically, they looked at two separate datasets, the first included 41 developmental events from 14 species of vertebrates, and the second included 116 developmental events from 14 mammal species and two amniote outgroups. Developmental events represented developmental transitions such as the first appearance of a structure (e.g., heart primordia) or some morphogenetic movement (e.g., fusion of the neural folds). The authors argued that analysis of developmental timing variation is valid for testing the hourglass model for two main reasons. The first is that variation in developmental timing provides an index of character linkage, as such variation depends on the dissociation of developmental events. The second reason is based on the predicted phenotypic similarities between species during the phylotypic stage, which according to the hourglass model occurs at mid-development. They argue that shifts in developmental timing will produce phenotypic differences, and therefore, if the hourglass model is correct, there should be minimal variation in developmental timing in the middle of the developmental sequence. However, developmental timing variation was greatest at mid-development for both the vertebrate and mammalian datasets, the opposite pattern to that predicted by the developmental hourglass model. Therefore, the authors concluded that vertebrates do not display a phylotypic stage.

Using molecular rather than morphological data, Roux and Robinson-Rechavi (2008) also provided evidence that brings the existence of the vertebrate phylotypic stage and the hourglass model into question. The authors argue that the phylotypic stage can be determined by the degree of developmental constraint as measured by the effects of directed knockout mutations, transgenic insertions, point mutations, and morpholinos. If the hourglass model applies to vertebrate development, then the effects of these mutations will be greatest at mid-development, representing the constricted portion of the hourglass. To test their hypothesis, Roux and Robinson-Rechavi used gene expression data from mice and zebrafish. Gene expression in mice was measured using EST counts for 26 stages of development, and in zebrafish from DNA microarrays over 14 stages of development. Instead of finding the greatest degree of developmental constraint at mid-development, flanked by less constraint earlier and later in development as predicted by the hourglass model, they found that the greatest amount of constraint occurs at the beginning of development. As development proceeds in both zebrafish and mice, the degree of developmental constraint steadily decreases. The authors suggest their results indicate that the hourglass model does not apply to vertebrate development. 
Contrary to the three studies discussed above, results from recent molecular studies suggest that the phylotypic stage does exist and that the hourglass model is valid. For example, in the first use of molecular data to test for the phylotypic stage, Hazkani-Covo et al. (2005) found evidence for the hourglass model in mouse embryos. Their dataset was comprised of over 1,500 mouse genes and their human orthologs that are expressed in over 26 stages of embryonic development. They measured the evolutionary divergence between corresponding orthologous proteins under the prediction that orthologs expressed during middevelopment will resemble each other more closely than during earlier or later stages (representing the hourglass model). While their results were far from conclusive, they did suggest that there is evidence for the vertebrate hourglass model in mice. Human and mouse ortholog expression was most similar between the first somites stage and the formation of the posterior neuropore. This developmental timeframe approximates the morphologically defined phylotypic stage in vertebrates.

Using a different molecular approach, Irie and SeharaFujisawa (2007) also provide evidence for the vertebrate phylotypic stage. The authors evaluated the expression of conserved genes among vertebrates at different stages of development using a mouse model. Under the assumption that strong developmental constraints occur during the phylotypic stage, they predict that genes conserved among vertebrates would be highly constrained during the phylotypic stage. Their results indicated a highly conserved embryonic period at days 8.0-8.5. Morphologically, this period in mice is marked by the appearance of the pharyngeal arches and somites and corresponds to the vertebrate phylotypic stage.

While the search for the phylotypic stage has generally been focused on vertebrates, Kalinka et al. (2010) used Drosophila species to determine if insects display a phylotypic stage. Specifically, they used DNA microarrays to measure genome-wide gene expression in six separate Drosophila species throughout the course of development. They predicted that variation in morphological patterning might be reflected by variation in gene expression. Therefore, they compared the timing of gene expression across all six species. The authors found that the variation in gene expression timing was least around the extended germ band stage, which corresponds to the insect phylotypic stage and supports the developmental hourglass model.

Despite these conflicting results, I agree with Hall (1997) that our current conception of the body plan and phylotypic stage is valid. Hall (1997) suggests that many arguments against the existence of the phylotypic stage are based on heterochronic (or timing) shifts in development, not morphological shifts. He compares the different mechanisms that lead to the phylotypic stage with the different mechanisms that lead to the gastrula among species. That the gastrula exists as a morphological entity is not questioned no matter how gastrulation is accomplished. Thus, Hall (1997) argues that there is no cause to abandon the body plan concept and the phylotypic stage simply because the developmental mechanisms that produce such conserved features differ among organisms.

Additionally, each of the studies discussed above uses different methods and criteria to determine the existence of the phylotypic stage. Comparisons among results are impossible given the differences in the studies. What is clear is that we must separate patterns from processes when we discuss the body plan concept. As Hall (1996) states so succinctly "Baupläne represent fundamental, structural and phylogenetic organization that is maintained despite variation in the developmental processes producing the structures." That conserved morphological patterns exist is clear. In fact, one of the most extraordinary evolutionary events known - the Cambrian explosion-provides evidence for the existence of body plans and marks the initial appearance of all animal forms.

\section{What Is the Cambrian Explosion?}

Fossilized animals first appear between approximately 550 530 million years ago (Conway Morris 2006). This period possibly represents the greatest evolutionary transition known, as representatives of all modern phyla appear at this time (Marshall 2006). That is, examples of all extant animal body plans arose within a 20 million-year time span (Conway Morris 2000, 2006; Erwin et al. 2011; Marshall 2006; Newman and Bhat 2009). This evolutionary event is therefore referred to as the Cambrian explosion, as it occurred during the Cambrian period and the appearance of these body plans is relatively sudden (Marshall 2006). Twenty million years is arguably not that sudden; however, when contrasted with the more than 500 million years that have passed since the first appearance of fossilized animals, and that no new phyla have appeared since, the Cambrian explosion was relatively sudden and explosive (Marshall 2006; Raff 1996).

Several researchers have expressed doubt that all animal body plans evolved in the 20 million years encompassed by the Cambrian explosion. Rather, they suggest that body plans arose in the Ediacaran and that fossilization potential evolved in the Cambrian, leading to the illusion of the sudden evolution of all animal phyla (Conway Morris 2000, 2006; Erwin et al. 2011; Marshall 2006). While the timeline for the initial appearance of phyla is controversial, that no new phyla have emerged in over 500 million years is generally accepted (though see Fitch and Sudhaus 2002). Why no new phyla have emerged in the time since the 
Cambrian explosion is one of biology's great mysteries. Five major extinction events have occurred since the explosion, offering vacated niches for new body plans to evolve. Still, no new phyla have been observed. For evolutionary developmental biologists, two main questions emerge from this phenomenon: (1) what developmental processes underlie this apparent phylum-level stasis and (2) what developmental processes underlie the evolution of novel features at the class, family, and species levels. The answers to these questions are generally sought under the concepts of constraint and evolvability respectively.

\section{Constraint}

Constraints can essentially be defined as the limited or channeled generation of variation (Maynard Smith et al. 1985). We recognize such constraints by the disparity in the types of creatures we can imagine and the types that actually exist. For example, we can read and write about griffins, satyrs, and mermaids, but we know that we will never actually see them in nature (Weiss and Buchanan 2009). Such body plans are not possible as a consequence of constraint.

In order for selection to act upon an organism, there must be variation. Therefore, as constraint refers to the limited or channeled production of variation, the range of possible responses to selection an organism can display are determined by constraint (Hall 1999). An important aspect of constraint is that it not only limits how much variation is generated, but it also channels the direction of variation generated (Hallgrímsson et al. 2012; Hendrikse et al. 2007). Clearly, much variation exists, as can be seen by comparing horses with turtles, birds, and starfish. Variation exists in multiple directions to allow for such morphological disparity, but it does not exist in every direction. And it is this channeling in the direction of variation that enables some animals to have wings, or shells or hooves, but does not allow for a half-human, half-goat to develop.

Constraints are generally thought to arise to ensure that certain structures develop properly. Many of the structures that vary the least in their development (i.e., most constrained) are found to be essential for survival. For example, Galis and Metz (2001) found that organisms exposed to teratogens during the phylotypic stage developed numerous abnormalities resulting in high mortality. The correlation between the development of certain structures and survival is thought to be caused by a relatively large number of inductive interactions between developmental processes (Galis and Metz 2001; Hall 1999; Riedl 1977). Due to numerous interactions with other developmental processes, disruption of central developmental processes (or those that occur during the phylotypic stage) will have widespread effects and thus affect survival. Therefore, to ensure proper development, some processes are constrained in their ability to vary, and this constraint is often maintained by stabilizing selection (Hall 1999).

While some structures have essentially remained unchanged for millions of years, suggesting constraints, other traits have changed dramatically and in a relatively short period of time. Structures exhibiting greater diversity have been able to produce more variation on which selection can act and can be described as being more evolvable. Understanding what makes a trait or process more evolvable is one of the main focal points of current evo-devo research (Hendrikse et al. 2007).

\section{Evolvability}

Although there are several definitions of evolvability as described by Pigliucci (2008), I follow the lead taken by Pavlicev and Wagner (2012) and use the definition set out by Lee Altenberg. He defined evolvability as "the ability of a population to produce variants fitter than any yet existing" (Altenberg 1994, p. 47). At first glance, evolvability seems to be constraint's opposite. While constraint limits the production of variation, evolvability is associated with the generation of variation. However, evolvability is a relational term, such as solubility, and therefore, the concept of evolvability applies equally to all traits whether highly or loosely constrained. Constrained traits are described as having low evolvability, whereas variable traits are considered to have high evolvability.

Given that many developmental processes are deeply entrenched within a species, class, phylum, or other taxonomic grouping, evolvability often requires the freeing of development from constraint. But what aspects of the developmental system allow for breaking from constraint? Budd (2006) outlines three properties of developmental systems that can free developmental systems from constraint and generate variation: functional asymmetries, redundancy, and preadaptation.

Functional asymmetry is based on Riedl's burden concept (see below) whereby some structures are more integrated within a system than others. As discussed above under constraints, structures with fewer interconnections are under less constraint and thus, are more variable. Selection can act on this variation to create change. Budd refers to differences in response to selection caused by differences in the degree of connectedness as functional asymmetry and suggests that this asymmetry introduces evolutionary flexibility into the system.

In evo-devo, redundancy is often described in terms of genes. For example, genetic redundancy describes the situation where two or more genes have overlapping functions 
and can either fully or partially substitute for each other (Thomas 1993; Wagner 1999, 2005). However, the concept of redundancy can be applied to any developmental process where overlap between components of the developmental system can substitute for each other, potentially freeing one or more of the components to take on new functions. Budd's description of redundancy is focused on functional overlap (Budd 2006). He further differentiates redundancy into actual and potential redundancy. When two or more components actually share a common function, they are described as actually redundant. Potential redundancy implies that under the right circumstances, two or more components could share a common function. Potential redundancy is related to the third property of developmental systems described by Budd, preadaptation.

Preadaptation refers to the phenomenon of a character acquiring a new function based on its previous or existing functionality. This change in function requires that characters are in the "right place at the right time," and they must display actual or partial redundancy with the new function (Budd 2006, p. 618). An example of preadaptation provided by Budd (2006) is the change in function developed by feathers. Initially, feathers were used as insulators or for display, but they have since acquired the additional function of flight devices.

The initial appearance of body plans, as well as the diversification of traits among species, families, and classes required evolvability of the developmental system. Yet, if we accept Altenberg's definition (1994) of evolvability, the variants that are produced through flexibility in the system must be more viable than those that already exist. The developmental system must therefore limit to some extent the direction, timing, and amount of variation that can be introduced. Thus, our modern conception of how body plans first arose, their stability over the last 500 million years, and the diversity introduced in later stages of development involves the interplay of constraint and evolvability.

\section{Body Plans and the Interplay Between Constraint and Evolvability}

As stated by Brigandt, "developmental constraint and morphological evolvability are two sides of one coin" (Brigandt 2007, p. 710). This view is similarly presented by Hall (1999) and Hallgrímsson et al. (2012), whereby morphological stability and change are driven by the same developmental and evolutionary mechanisms but in different combinations. Another theme in the modern conception of the body plan is that both adaptive and developmental principles are incorporated (Brigandt 2007; Hallgrímsson et al. 2012). Traditionally, evolutionary theory focused on the interactions between phenotypic variation, selection, and drift, thereby accounting for small modifications of existing structures (Muller and Newman 2005). Thus, evolutionary theory accounts for microevolutionary changes. The origin of entirely new traits, or macroevolutionary changes, fell under a different research program such as developmental evolution (Hallgrímsson et al. 2012; Muller and Newman 2005; Schlosser and Wagner 2004; Wagner and Mezey 2004). As described by Hallgrímsson et al. (2012), marrying adaptationism and development provides an explanation for all evolutionary change, big or small. The first person to bring together these two sets of seemingly contrasting concepts (constraint and evolvability) and mechanisms (adaptation and development) was Rupert Riedl.

Riedl brought the body plan concept from its largely metaphysical definition to its current position as a variational concept (Wagner and Laubichler 2004). He noted that the evolutionary patterns we observe, particularly major changes such as the origin of body plans, are more structured than predicted by traditional Neo-Darwinian theory based on population genetics and adaptation (Riedl 1977, 1978). Thus, he reasoned that there is some other underlying mechanism or mechanisms that account for evolutionary change. Instead of abandoning traditional evolutionary theory, Riedl suggested that development structures new variation and that it is this structured variation that is available to selection (Wagner and Laubichler 2004). In this way, Riedl integrated developmental and Neo-Darwinian, or adaptive approaches, to evolutionary change.

A main pattern of evolutionary change noticed by Riedl was that different characters evolve at markedly different rates and that traits associated with body plans evolve particularly slowly (Wagner and Laubichler 2004). To explain this phenomenon, Riedl introduced two concepts: burden and evolvability. Burden describes the probability of character evolution based on the importance of that character's function(s) and the number of other traits that depend on the character in question. A trait has a high burden if many other traits depend on it and if its function is of great importance. Traits with a high burden evolve very slowly. Conversely, a trait with few or no dependent traits, and of minor importance, will have little burden and is free to evolve more rapidly (Riedl 1977; Schoch 2010; Wagner and Laubichler 2004).

An important aspect of the burden concept is that burdens are hierarchically nested. As new characters are added, they emerge in the context of the pre-existing characters (Wagner and Laubichler 2004). New characters are dependent on preexisting traits, thus increasing the amount or rank of the burden of the older traits. In this way, the burden of a trait is considered by Riedl to be directly correlated with its phylogenetic age (Schoch 2010; Wagner and Laubichler 2004). This relationship demonstrates how Riedl incorporated 
development with Neo-Darwinian ideas of slow gradual evolution. Body plan characters do not arise as body plan characters. Rather, they emerge as novel traits that are not yet entrenched within a species or highly conserved. These new traits are dependent on the traits that came before them, and with time and the addition of new traits, their burden increases until they are essentially fixed.

Riedl's related concept, evolvability, refers to the probability that a new trait arising from mutation will be viable (Wagner and Laubichler 2004). A trait with high evolvability has a relatively high probability for change that will lead to viable results. Riedl noted, however, that most mutations are deleterious and will therefore be selected against. Contrary to what one might initially expect, burden can actually increase the evolvability of a trait. Burden helps to structure variation, channeling emerging variation into directions that are more likely to produce viable phenotypes (Riedl 1977; Wagner and Laubichler 2004). Again, this concept bridges developmental and Neo-Darwinian aspects of evolutionary change. Burden, built into the developmental architecture of a trait or an organism, helps to channel variation that is available to natural selection to produce adaptive phenotypes (or increase evolvability). Therefore, heavily burdened traits, such as body plan characters, provide a developmental framework which can increase the evolvability of other traits within an organism.

Schoch (2010) outlines how Riedl's body plan concept matches our current conception of the idea given the advancements in evolutionary biology. He suggests that Riedl was likely correct in his claim that body plan characters arise stepwise in a gradual manner, and that he was right that body plan characters become increasingly constrained due to internal selection. However, as has been pointed out by several authors, constraints have been found to be breakable (Budd 2006; Maynard Smith et al. 1985; Schwenk and Wagner 2003), and it is therefore unlikely that overcoming body plan constraints is impossible (Schoch 2010). As noted by Meatloaf, "two out of three ain't bad."

By integrating developmental and Neo-Darwinian evolutionary approaches to the study of body plans, Riedl is largely responsible for bringing the body plan concept from its metaphysical past to its current position at the center of evo-devo research. While Riedl may have missed the mark on a few details, much of his conception of burden, evolvability, and body plans is proving relevant in current studies of genetic regulatory networks (GRNs).

\section{Riedl's Burden and Evolvability Concepts and Their Relevance to Genetic Regulatory Networks}

Much as Riedl had noted that at an organismal level most mutations would lead to deleterious, unviable phenotypes, a similar observation has been made at the level of gene regulatory networks by Davidson and Erwin $(2006,2010)$ and Erwin and Davidson (2009). If gene networks are unstructured, or essentially random, then any small change within the network will likely lead to disastrous results, as changes will spread throughout the network easily. Following from Riedl's idea of nested burden, Davidson and Erwin (2006) proposed that GRNs are structured by their inherent hierarchical modular composition. They discuss four types of modules within GRNs: kernels, plug-ins, input/output (I/O) switches, and gene batteries.

Kernels are inflexible subcircuits of approximately three to eight genes that perform essential functions such as developmental patterning and specification of the spatial domain of a given body part. Disruption of any gene within a kernel causes catastrophic phenotypic results, and therefore, kernel structure is highly conserved (Davidson and Erwin 2006). In Riedl's terms, kernels are highly burdened and are responsible for characters that make up body plans. Moving down the GRN hierarchy come the plug-ins, which, like kernels, consist of structurally conservative subcircuits of regulatory genes. Plug-ins are used for many different developmental functions and are shared among species. Unlike kernels, plug-ins are not dedicated to the development of specific body parts; rather, their deployment is flexible and used in diverse networks (Davidson and Erwin 2006). I/O switches often consist of cis-regulatory linkages that help to regulate other network subcircuits. I/O switches fall further down the GRN modular hierarchy and allow for greater evolutionary change of developmental processes. That is, I/O switches are less burdened than plug-ins, which are in turn less burdened than kernels. The least burdened of the four modules are gene batteries. While kernels are central to the entire gene network, the products of gene batteries are the terminal outputs of GRNs, and thus, batteries are found at the periphery of GRNs. They are protein-coding genes that produce proteins that help perform cell type-specific functions. Unlike kernels and plug-ins, gene batteries are not regulatory and as such are able to evolve relatively quickly (Davidson and Erwin 2006).

Further molecular evidence for Riedl's concepts of burden, evolvability, and body plans is described by $\mathrm{He}$ and Deem (2010). Using the gene networks described by Davidson and Erwin (2006), He and Deem measured the ratio of nonsynonymous substitution to synonymous substitution of the genes within the networks (a widely accepted measure of the rate of evolution). Their results indicate that genes evolve at different rates and that these rates correspond to the hierarchical module in which genes are found. That is, genes within plug-ins evolve more quickly than genes within kernels, genes within I/O switches evolve more quickly than genes within plug- 
ins, and that genes within batteries evolve the most quickly.

The hierarchical structure of GRNs provides a mechanistic explanation for the evolutionary patterns we observe at the organismal level, such as some traits that are highly conserved (body plan characters) and some traits that vary greatly among organisms. The highly conserved kernels within GRNs form characters that might be shared at the phylum level, whereas plug-ins and I/O switches determine traits shared at the class and family levels, and batteries produce traits shared at the species level (He and Deem 2010). As with Riedl's burden and evolvability concepts, a modular GRN structure allows for evolutionary changes to occur at the peripheries of the network (within gene batteries) without disturbing the essential regulatory functions executed at the core of the network by kernels. The somewhat constraining nature of structured GRNs actually increases the probability that changes - at least at the periphery of networks - will be viable.

\section{Conclusions}

The body plan concept is not the archaic, outdated, metaphysical concept that it is described to be by some researchers. Rather, it is based on modern evolutionary principles and provides a conceptual foundation for evo-devo research. Major evo-devo concepts such as constraint, evolvability, homology, canalization, integration and modularity, heterochrony, and other concepts are based on the existence of animal body plans. While there is ongoing scientific debate about specific aspects of the body plan concept, such as the phylotypic stage and the developmental hourglass model, the existence of body plans, their relatively rapid emergence, and their conservation since they first appeared are not disputed. Additionally, the body plan concept is an active area of research in its own right, drawing from the latest advancements in genetics and computer modeling. Among the most interesting and fundamental unanswered questions in biology are: (1) how did animal body plans first arise and (2) how have animal body plans remained essentially unchanged since their emergence. Answers to these questions, or at least partial answers, will be found through research on body plans.

As technological advancements allow for more indepth molecular, morphological, and computer modeling analyses, our understanding of body plans will continue to deepen and change. Given the centrality of the body plan concept in evo-devo research, it is important that researchers in the field are up to date on our current conception of the body plan and its implications for their own studies.
Acknowledgments I would like to thank Niles, Michelle, and Greg Eldredge for the opportunity to assemble and contribute to this special issue. I greatly appreciate their support, guidance, and patience. I am also grateful to Quentin Cronk and Brian Hall for their helpful insights on various aspects of this paper. I thank Melissa Cooper for her helpful comments on an earlier draft of this paper.

\section{References}

Altenberg L. The evolution of evolvability in genetic programming. In: Kinnear Jr KE, editor. Advances in genetic programming. Cambridge: MIT Press; 1994. p. 47-74.

Appel TA. The Cuvier-Geoffroy debate: French biology in the decades before Darwin. New York: Oxford University Press; 1987.

Atkinson JW. Conceptual issues in the reunion of development and evolution. Synthese. 1992;91:93-110.

Ballard WW. Morphogenetic movements and fate maps of vertebrates. Am Zool. 1981;21:391-9.

Bininda-Emonds ORP, Jeffery JE, Richardson MK. Inverting the hourglass: quantitative evidence against the phylotypic stage in vertebrate development. Proc R Soc Lond B. 2003;270:341-6.

Brigandt I. Typology now: homology and developmental constraints explain evolvability. Biol Philos. 2007;22:709-25.

Budd GE. On the origin and evolution of major morphological characters. Biol Rev. 2006;81:609-28.

Carlson BM. Human embryology and developmental biology. 4th ed. Philadelphia: Elsevier; 2009.

Conway Morris S. Nipping the Cambrian "explosion" in the bud? Bioessays. 2000;22:1053-6.

Conway Morris S. Darwin's dilemma: the realities of the Cambrian 'explosion'. Phil Trans R Soc B. 2006;361:1069-83.

Cronk QCB. Plant evolution and development in a post-genomic context. Nat Rev Genet. 2001;2:607-19.

Davidson EH, Erwin DH. Gene regulatory networks and the evolution of animal body plans. Science. 2006;311:796-800.

Davidson EH, Erwin DH. Evolutionary innovation and stability in animal gene networks. J Exp Zool (Mol Dev Evol). 2010;314B:1826.

de Beer GR. Embryos and ancestors. Oxford: Clarendon Press; 1940.

de Beer GR. Embryos and ancestors. Oxford: Oxford University Press; 1958.

Duboule D. Temporal colinearity and the phylotypic progression: a basis for the stability of a vertebrate bauplan and the evolution of morphologies through heterochrony. Development (Suppl); 1994. pp. 135-142.

Eldredge N. Macroevolutionary dynamics: species, niches and adaptive peaks. New York: McGraw-Hill; 1989.

Elinson RP. Changes in developmental patterns: embryos of amphibians with large eggs. In: Raff RA, Raff EC, editors. Development as an evolutionary process. New York: Liss; 1987. p. 1-21.

Erwin DH, Davidson EH. The evolution of hierarchical gene regulatory networks. Nat Rev Genet. 2009;10:141-8.

Erwin DH, Laflamme M, Tweedt SM, Sperling EA, Pisani D, Peterson KJ. The Cambrian conundrum: early divergence and later ecological success in the early history of animals. Science. 2011;334:1091-7.

Fitch DHA, Sudhaus W. One small step for worms, one giant leap for "Bauplan?". Evol Dev. 2002;4:243-6.

Galis F, Metz JAJ. Testing the vulnerability of the phylotypic stage: on modularity and evolutionary conservation. J Exp Zool. 2001;291:195-204.

Garstang W. The theory of recapitulation. A critical restatement of the biogenetic law. J Linn Soc Lond (Zool). 1922;35:81-101. 
Gould SJ. Ontogeny and phylogeny. Cambridge: Harvard University Press; 1977.

Gould SJ. Wonderful life: the Burgess Shale and the nature of history. New York: Norton; 1989.

Graham LE, Cook ME, Busse JS. The origin of parts: body plan changes contributing to a major evolutionary radiation. PNAS. 2000;97:4535-40.

Haeckel E. Anthropogenie oder Entwickelungsgeschichte des Menschen. Leipzig: Englemann; 1874.

Hall BK. Baupläne, phylotypic stages and constraint: why there are so few types of animals. Evol Biol. 1996;29:251-61.

Hall BK. Phylotypic stage or phantom: is there a highly conserved embryonic stage in vertebrates? TREE. 1997;12:461-3.

Hall BK. Evolutionary developmental biology. 2nd ed. Dordrecht: Kluwer; 1999.

Hallgrímsson B, Jamniczky HA, Young NM, Rolian C, Schmitt-Ott U, Marcudio RS. The growth of variation and the developmental basis of evolutionary novelty. J Exp Zool (Mol Dev Evol); 2012 (in press).

Hazkani-Covo E, Wool D, Graur D. In search of the vertebrate phylotypic stage: a molecular examination of the developmental hourglass model and von Baer's third law. J Exp Zool (Mol Dev Evol). 2005;304B:150-8.

He J, Deem MW. Hierarchical evolution of body plans. Dev Biol. 2010;337:157-61.

Hendrikse JL, Parsons TE, Hallgrímsson B. Evolvability as the proper focus of evolutionary developmental biology. Evol Dev. 2007:9:393-401.

Irie N, Sehara-Fujisawa A. The vertebrate phylotypic stage and an early bilaterian-related stage in mouse embryogenesis defined by genomic information. BMC Biol. 2007. doi:10.1186/1741-70075-1.

Kalinka AT, Varga KM, Gerrard DT, Preibisch S, Corcoran DL, Jarrells $\mathrm{J}$, et al. Gene expression divergence recapitulates the developmental hourglass model. Nature. 2010;468:811-4.

Kardong KV. Vertebrates: comparative anatomy, function, evolution. 4th ed. Boston: McGraw-Hill; 2006.

Lillie FR. The development of the chick. New York: Holt; 1919.

Marshall CR. Explaining the Cambrian "explosion" of animals. Annu Rev Earth Planet Sci. 2006;34:355-84.

Maynard Smith J, Burian R, Kauffman S, Alberch P, Campbell J, Goodwin B, et al. Developmental constraints and evolution. Quart Rev Biol. 1985;60:265-87.

Mayr E. The growth of biological thought. Cambridge: Harvard University Press; 1982.

Moore KL, Persaud TVN. The developing human: clinically oriented embryology. 8th ed. Philadelphia: Elsevier; 2008.

Muller GB, Newman SA. The innovation triad: an EvoDevo agenda. J Exp Zool (Mol Dev Evol). 2005;304B:487-503.

Newman SA. Animal egg as evolutionary innovation: a solution to the "embryonic hourglass" puzzle. J Exp Zool (Mol Dev Evol). 2011;316B:467-83.

Newman SA, Bhat R. Dynamical patterning modules: a "pattern language" for development and evolution of multicellular form. Int J Dev Biol. 2009;53:693-705.

Nielsen C. Defining phyla: morphological and molecular clues to metazoan evolution. Evol Dev. 2003;5:386-93.

Panchen AL. Etienne Geoffroy St.-Hilaire: father of "evo-devo"? Evol Dev. 2001;3:41-6.

Pavlicev M, Wagner GP. Coming to grips with evolvability. Evol Edu Out; 2012 (in press).

Pigliucci M. Is evolvability evolvable? Nat Rev Genet. 2008;9:75-82.

Raff RA. The shape of life: genes, development, and the evolution of animal form. Chicago: The University of Chicago Press; 1996.

Richardson MK. Heterochrony and the phylotypic period. Dev Biol. $1995 ; 172: 412-21$
Richardson MK, Hanken J, Gooneratne ML, Pieau C, Raynaud A, Selwood L, et al. There is no highly conserved embryonic stage in the vertebrates: implications for current theories of evolution and development. Anat Embryol. 1997;196:91-106.

Riedl R. A systems-analytical approach to macroevolutionary phenomena. Quart Rev Biol. 1977;52:351-70.

Riedl R. Order in living organisms: a systems analysis of evolution. New York: Wiley; 1978.

Rieppel O. 'Type' in morphology and phylogeny. J Morphol. 2006;267:528-35.

Roux J, Robinson-Rechavi M. Developmental constraints on vertebrate genome evolution. PLoS Genetics. 2008. doi:10.1371/ journal.pgen. 1000311.

Rychel AL, Smith SE, Shimamoto HT, Swalla BJ. Evolution and development of the chordates. Coll Pharyngeal Cartil. 2006;23:541-9.

Sander K. The evolution of patterning mechanisms: gleanings from insect embryogenesis and spermatogenesis. In: Goodwin BC, Holder N, Wylie CC, editors. Development and evolution. Cambridge: Cambridge University Press; 1983. p. 137-60.

Schlosser G, Wagner GP. Introduction: the modularity concept in developmental and evolutionary biology. In: Schlosser G, Wagner GP, editors. Modularity in development and evolution. Chicago: The University of Chicago Press; 2004. p. 1-11.

Schoch RR. Riedl's burden and the body plan: selection, constraint, and deep time. J Exp Zool (Mol Dev Evol). 2010;314B:1-10.

Schwenk K, Wagner GP. Constraint. In: Hall BK, Olson WM, editors. Keywords and concepts of evolutionary developmental biology. Cambridge: Harvard University Press; 2003. p. 52-61.

Sedgwick A. On the law of development commonly known as von Baer's law; and on the significance of ancestral rudiments in embryonic development. Q J Microsc Sci. 1894;36:35-52.

Seidel F. Körpergrundgestalt und Keimstruktur. Eine Erörterung über die Grundlagen der vergleichenden und experimentellen Embryologie und deren Gültigkeit bei phylogenetischen Überlegungen. Zool Anz. 1960;164:245-305.

Slack JMW, Holland WH, Graham CF. The zootype and the phylotypic stage. Nature. 1993;361:490-2.

Thomas JH. Thinking about genetic redundancy. Trends Genet. 1993;9:305-9.

Valentine JW. Fossil record of the origin of Baupläne and its implications. In: Raup DM, Jablonski D, editors. Patterns and processes in the history of life. New York: Springer; 1986. p. 209-31. Dahlem Conference.

Von Baer KE. Entwicklungsgeschichte der Thiere: Beobachtung und Reflexion. Königsberg: Bornträger; 1828.

Wagner A. Redundant gene functions and natural selection. J Evol Biol. 1999;12:1-16.

Wagner A. Distributed robustness versus redundancy as causes of mutational robustness. Bioessays. 2005;27:176-88.

Wagner GP, Laubichler MD. Rupert Riedl and the re-synthesis of evolutionary and developmental biology: body plans and evolvability. J Exp Zool (Mol Dev Evol). 2004;302B:92-102.

Wagner GP, Mezey JG. The role of genetic architecture constraints in the origin of variational modularity. In: Schlosser G, Wagner GP, editors. Modularity in development and evolution. Chicago and London: The University of Chicago Press; 2004. p. 338-58.

Weiss KM, Buchanan AV. The mermaids tale: four billion years of cooperation in the making of living things. Cambridge: Harvard University Press; 2009.

Wolpert L. The triumph of the embryo. Oxford: Oxford University Press; 1991.

Woodger JH. On biological transformations. In: Le Gros Clark WE, Medewar PB, editors. Growth and form: essays presented to D’Arcy Thompson. Oxford: Clarendon Press; 1945. p. 95120. 